\title{
TV White Space Channel Allocation with Simulated Annealing as Meta Algorithm
}

\author{
Bo Ye \\ ESBE, London South Bank Uni. BT Research, Polaris 134 Adastral Park, \\ London SE1 0AA, UK
}

\author{
Anjum Pervez, and Mohammad Ghavami \\ ESBE, London South Bank Uni. \\ London SE1 0AA, UK
}

\begin{abstract}
When developing a TV White Space (TVWS) system with the available TV spectrum after digital switchover, channel allocation for TVWS devices to avoid interference becomes one of the most challenging problems. In this paper, Simulated Annealing (SA) is applied to solve the problem to minimize the total interference. However, the performance of SA applied to the problem depends on the appropriate choice of several key parameters. Hence, all parameters are listed and experiments with all possible combinations are done and the parameters that perform best are chosen manually. Manual selection requires a lot of experiments and is not effective. Therefore, an algorithm using SA as meta algorithm is proposed to choose the parameters automatically for SA. Finally the result of parameters selected automatically is compared with the result of those selected manually. We will show that automatic selection performs better compared with manual selection.
\end{abstract}

Index Terms-Simulated Annealing, Hyper-heuristics Algorithm, Channel Allocation, TV White Space.

\section{INTRODUCTION}

The proposed switchover from analogue to Digital TV (DTV) in the UK and elsewhere in Europe and the United States has generated particular interest in cognitive wireless networks strategies to find new solutions for Radio Resource Management. After the switchover has been completed, a significant amount of RF spectrum within the existing TV band which are also known as TV White Space (WS) will become vacant for sharing. It is strongly believed that the devices with cognitive capability will be the prime contenders for the dynamic spectrum access to TVWS. When developing a TVWS system, three methods of sensing, beacons and Geolocation combined with database are used to avoid interference between TVWS devices and the primary TV band users. In sensing, TVWS devices detect the presence of TV signals and use the spectrum not used by the primary TV band users. In systems using beacons, devices transmit when they receive a beacon signal and the information of available channels. In our project, Geo-location is used to allocate available spectrum at a certain time in a given position. In Geo-location systems, all devices including TV transmitters and TVWS devices will register in a database and send the information of position, power and so on to the database.

Furthermore, TVWS devices ideally should not cause interference to their adjacent devices. The challenge is managing co-channel interference. For a large network with lots of devices, allocating the channels among all devices to minimize the total interference is an NP-complete problem. Briefly, this means that globally optimal solutions to these problems cannot be found because the computing time required to do so grows exponentially with the problem size. Hence, heuristic methods must be used, which find solutions that are close to optimal in a tractable amount of time.

A distributed channel allocation was proposed in [1] based on Simulated Annealing (SA). Authors developed a channel allocation scheme to minimize the total interference of all Access Points (AP) in WLAN. In [1], authors proposed a distributed SA to solve the dynamic channel allocation problem in $802.11 \mathrm{~b} / \mathrm{g}$ based HD-WLANs. When using SA, authors did not mention the temperature initialization, the details of choosing cooling schedule. And they did not give any other methods of generating neighbor state.

Genetic Algorithm (GA) is a search heuristic that mimics the process of natural evolution. This heuristic is routinely used to generate useful solutions to optimization and search problems. GAs belong to the larger class of Evolutionary Algorithms (EA), which generates solutions to optimization problems using techniques inspired by natural evolution, such as inheritance, mutation, selection, and crossover. In [2, 3, 4, 5], GA is used to find a channel allocation from a search space to satisfy some constraints. In [6], authors proposed a fully distributed and self-managed algorithm. In [7], the channel assignment problem is solved by a neural network algorithm.

In [8], authors proposed a hybrid algorithm that combines GA and SA. The algorithm will trap in local minima, because in the mutation step, authors allow the selected AP to choose the channel used least among its neighboring APs. With such a choice, the search strategy moves to the domain of local search, and the mutation used to jump out the local minima loses its original meaning.

$\mathrm{SA}$ is a powerful technique for finding near-optimal solutions to NP-complete problem. However, SA relies on parameters that have to be fixed before the execution of the algorithm. It is usually very hard to choose appropriate parameters. A good choice of the parameters depends on the particular problem. Determining a good parameter setting often requires the execution of lots of time-consuming experiments with the aggravation that the best parameter setting for one problem is of limited utility for any other problem. In this paper, our contribution is to list all the parameters which involve our own approach, to list most of classic methods of the 


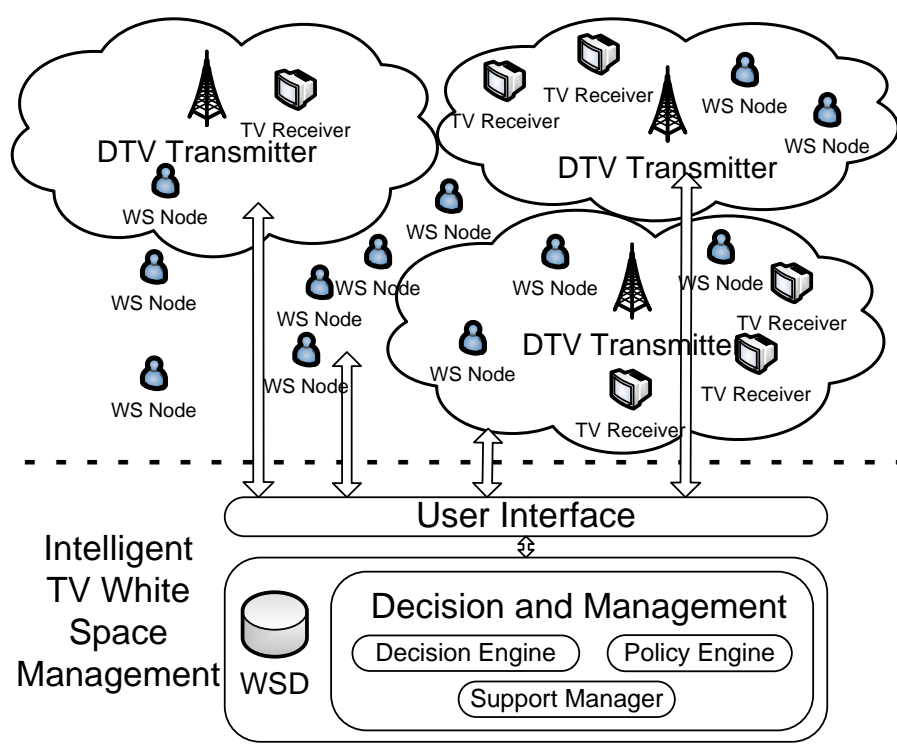

Fig. 1. The architecture of TVWS network

parameters and to investigate all possible combinations to find the ones performing best. Here SA with SA as meta algorithm is proposed to select the parameters of SA automatically.

The paper is organized as follows. In section II, system model and detailed problem are introduced. In section III, all parameters of SA are introduced. In section IV, simulation results comparing different possible combinations of parameters are presented and parameters are selected manually. The algorithm using SA as meta algorithm to select parameters automatically is introduced in section $\mathrm{V}$. Conclusions are given in section VI.

\section{System Model}

In Fig.1, our project Intelligent TVWS Management consists of three main modules: User Interface, White Space Database (WSD) and Decision \& Management (DAM).

In WSD, information about availability of spectrum (presence or absence of white spaces), location, transmission signal, mobility, power, and so on are also stored and updated dynamically; DAM is to make intelligent decisions and manage/verify information; Decision Engine (DE) performs the decision process and optimizes the result when users sending their requests; Support Manager (SM) translates the radio environment, verifies position of TVWS devices and ensures level of security; Policy Engine (PE) checks the validity of the decision made by the DE, and ensures that the decisions conform to the regulations, standards and specifications. PE may also guide DE to make valid decisions.

In our project, based on the information of DTV Transmitters, the information about availability of spectrum are calculated and then stored in WSD when the system deployed. The flow of processing users' requests is shown in Fig. 2. In the following part we further elaborate on the algorithm of reallocating channels. When simulating, we assume that in WSD channels 51-59 of TVWS [9] can be used and three

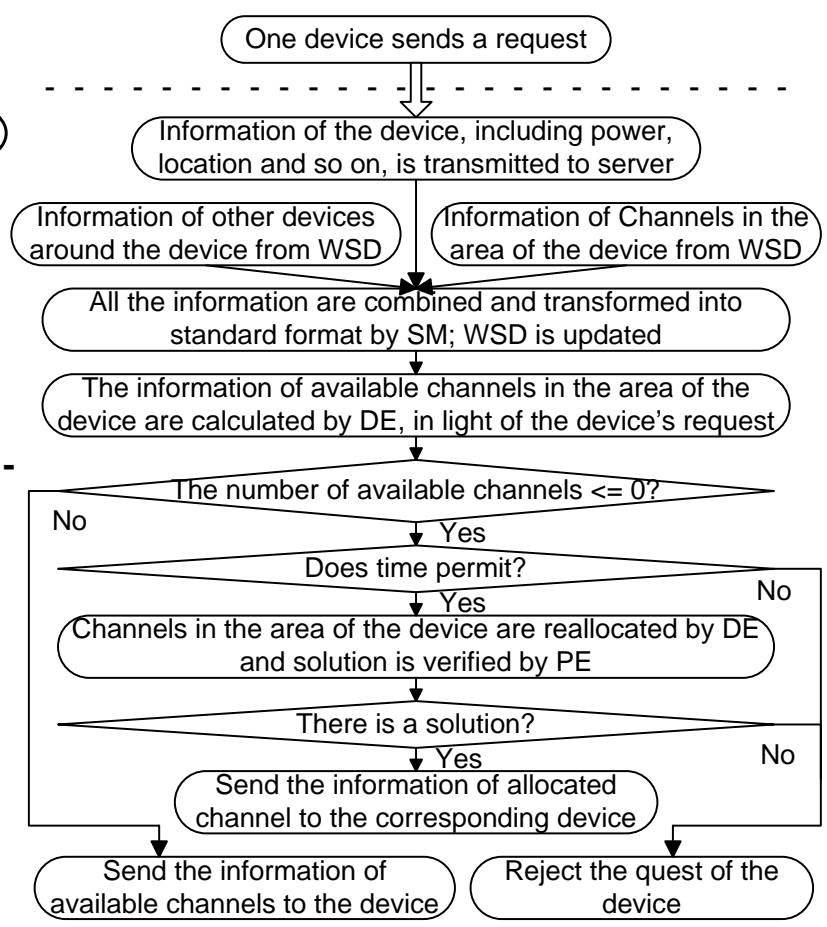

Fig. 2. The flow chart of processing the request of one device

channels are used as one. So channels 710-734, 734-758, 758$782(\mathrm{MHz})$ can be allocated. The middle frequencies 722,746 , $770 \mathrm{MHz}$ of the interval are used to stand for the channels.

$$
P_{i j}(d)=P_{j} G_{j} G_{i}\left(\lambda /\left(4 \pi d_{i j}\right)\right)^{2} / L
$$

Equation (1) is used to define the propagation loss. $P_{i j}(d)$ is the received power which is a function of the $i-j$ separation. $P_{j}$ is the power of transmitter $j . G_{j}$ is the transmitter $j$ antenna gain. $G_{i}$ is the receiver $i$ antenna gain. $d_{i j}$ is the $i-j$ separation distance in meters. $L$ is the system loss factor not related to propagation ( $L \geq 1)$. $\lambda$ is the wavelength in meters.

The goal is to minimize the total interference among WS devices. Channel allocation array $X$ is defined as $X=$ $\left\{x_{1}, x_{2}, x_{3}, \ldots \ldots, x_{n}\right\}$, where $x_{i} \in\{1,2,3\}$ ( 1 for $722 \mathrm{MHz}$, 2 for $746 \mathrm{MHz}, 3$ for $770 \mathrm{MHz}), i \in\{1,2,3, \ldots \ldots, n\}$.

In equation (2), when $i$ and $j$ use the same channel, the interference caused by device $j$ to $i I_{i j}\left(x_{i}, x_{j}\right)$ is calculated from (1). If they use different channels, it takes 0.

$$
I_{i j}\left(x_{i}, x_{j}\right)= \begin{cases}P_{i j} & x_{i}=x_{j} \\ 0 & x_{i} \neq x_{j}\end{cases}
$$

The objective function (3) is the sum of the interference of all devices. $\sum_{j=1}^{n} I_{i j}\left(x_{i}, x_{j}\right)$ is the sum of all the interference caused by other devices to $i$.

$$
\operatorname{Min} I(X)=\sum_{i=1}^{n} \sum_{j=1}^{n} I_{i j}\left(x_{i}, x_{j}\right) \quad(1 \leq i, j \leq n \& j \neq i)
$$

Subject to $X=\left\{x_{1}, x_{2}, x_{3}, \ldots \ldots, x_{n}\right\} \quad x_{i} \in\{1,2,3\}$ 


\section{Parameters of Simulated Annealing}

Simulated Annealing is motivated by an analogy to annealing in solids. The idea of SA comes from a paper published by Metropolis etc al in 1953. The algorithm simulated the cooling of material in a heat bath. This is a process known as annealing. In this technique, the states of a physical system are generated to states of a system being optimized, the physical energy is generalized to the function being minimized, and the temperature is generalized to a control parameter for the optimization process.

SA works by simulating a random walk on the set of states. It searches the state space looking for low-energy state. In this paper, the states are the solutions of channel allocation among WS devices. A solution space $\Omega$ is the domain of the objective function (3). The steps of the simulation are followed.

1. $X \in \Omega$ is a current solution. Then a neighbor solution $X^{\prime}$ of the current solution $X$ is selected.

2. Equation (4) is used to determine if $X$ is replaced by $X^{\prime}$ or not. $\mathrm{T}$ is a temperature controlling the probability of downward steps.

$$
\begin{aligned}
& \triangle I=I\left(X^{\prime}\right)-I(X) \\
& X=X^{\prime} \text { if } \triangle I<0 \text { or } \\
& r<\operatorname{AcceptProbability~}(T, \triangle I)(r=\operatorname{random}(0,1))
\end{aligned}
$$

The following pseudo code is the bare-bones algorithm of SA used in this paper.

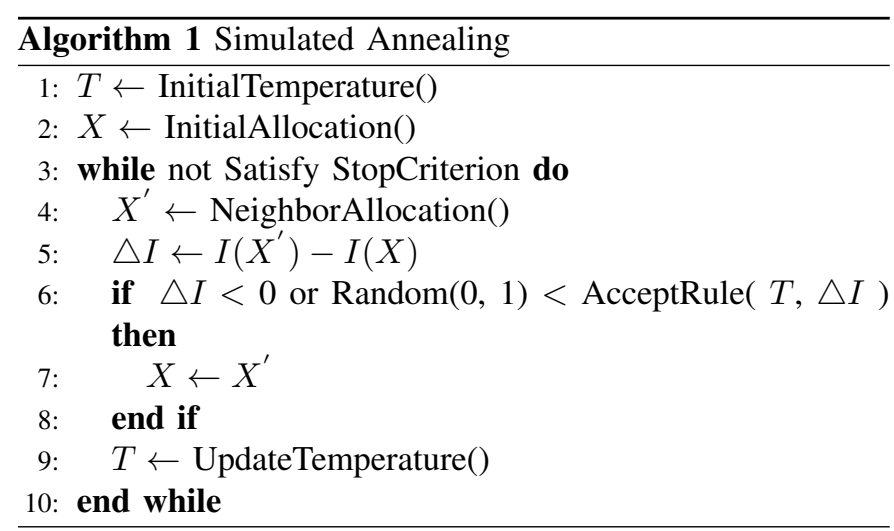

\section{A. Neighbor Allocation}

1) Neighbor Allocation with ASA (NAASA): In NAASA, Adaptive Simulated Annealing (ASA) [10] is used to generate neighbor allocations. $[A, B]$ is the range of $X$. In this paper, $u^{i} \in U[0,1] ; A=1$ and $B=3$ for $x_{i} \in[1,3]$.

$$
\begin{aligned}
& y^{i}=\operatorname{sgn}\left(u^{i}-1 / 2\right) T_{i}\left(\left(1+1 / T_{i}\right)^{\left|2 u^{i}-1\right|}-1\right) \\
& X^{\prime}=\operatorname{round}\left(X+y^{i}(B-A)\right)
\end{aligned}
$$

2) Neighbor Allocation at Random (NAR): In NAR, one device is selected at random and then a new channel is also selected at random to replace the old one of the device.

\section{B. Temperature Initialization}

1) Temperature Initialized by Acceptance Ratio (TIAR):

In TIAR, temperature is initialized so that the fraction of the solutions examined in the process of initialization will be accepted by acceptance rule, and there are two user-defined parameters: $n$ (Steps) is the number of the steps to determine initial temperature; $r \in[0,1]$ (Ratio) is the fraction of the solutions examined that will be accepted.

The objective function is followed. Bisection search is used to find value $T_{\text {init }}$ that satisfies acceptance ratio.

$$
\sum_{i=1}^{n} \operatorname{AcceptProbability}\left(T_{\text {init }}, \triangle I_{i}\right)=n r
$$

2) Temperature Initialized by Standard Deviation (TISD): In TISD, temperature is initialized with the user-defined ratio of standard deviation of interference $I(X)$ of the solutions examined [11]. There are also two parameters: $n$ (Steps) is the number of the steps to determine initial temperature; $r>0$ (Ratio) is the ratio of standard deviation.

$$
T_{\text {init }}=r \sqrt{\sum_{i=1}^{n}\left(I_{i}-\left(\sum_{i=1}^{n} I_{i}\right) / n\right)^{2} /(n-1)}
$$

\section{Acceptance Rules}

1) Metropolis Acceptance Probabilities (MAP): The following acceptance probability is used in Metropolis [11] algorithm.

$$
P=e^{-\triangle I / T_{\text {current }}}
$$

2) Fast Annealing Acceptance Probabilities (FAAP): The following acceptance rule is used in [12]'s fast annealing.

$$
P=1 /\left(1+e^{-\triangle I / T_{\text {current }}}\right)
$$

3) Tsallis Acceptance Probabilities (TSAP): Inspired by the success of fast annealing, the following method in [13] accepts large jumps occasionally.

$$
P= \begin{cases}1 & \triangle I \leq 0 \\ \left(1-\frac{(1-q) \triangle I}{T}\right)^{\frac{1}{1-q}} & \triangle I>0 \& \frac{(1-q) \triangle I}{T} \leq 1 \\ 0 & \triangle I>0 \& \frac{(1-q) \triangle I}{T}>1\end{cases}
$$

4) Optimality of Threshold Acceptance Probabilities $(O T A P):$ A modified Tsallis acceptance probability is introduced in [14].

$$
P= \begin{cases}1 & \triangle I \leq 0 \\ \left(1-\frac{(1-q) \triangle I}{(2-q) T}\right)^{\frac{1}{1-q}} & \triangle I>0 \& \frac{(1-q) \triangle I}{(2-q) T} \leq 1 \\ 0 & \triangle I>0 \& \frac{(1-q) \triangle I}{(2-q) T}>1\end{cases}
$$

5) Threshold Acceptance Probabilities (TAP): In [15], one method called threshold annealing is used to provide simple version by saving time to evaluate the exponential. The acceptance probability is followed.

$$
P= \begin{cases}1 & \triangle I \leq T \\ 0 & \triangle I>T\end{cases}
$$


TABLE I

RESUlts of COMBINATIONS WITH NAR, ES IN PROCEDURE 1

\begin{tabular}{|l|c|c|c|c|c|}
\hline & OTAP & MAP & FAAP & TAP & TSAP \\
\hline TIAR & 17 & 15 & 24 & 16 & 12 \\
\hline TISD & 64 & 55 & 59 & 58 & 48 \\
\hline
\end{tabular}

TABLE II

RESUlts of COMBINATIONS WITH TISD AND FAS IN PRocedure 1

\begin{tabular}{|c|c|c|c|c|c|}
\hline & OTAP & MAP & FAAP & TAP & TSAP \\
\hline NAASA & 57 & 59 & 66 & 64 & 51 \\
\hline NAR & 54 & 79 & 71 & 65 & 66 \\
\hline
\end{tabular}

TABLE III

RESULTS OF COMBINATIONS WITH TISD AND FAS IN PROCEDURE 2

\begin{tabular}{|c|c|c|c|c|c|}
\hline & OTAP & MAP & FAAP & TAP & TSAP \\
\hline NAASA & 40 & 58 & 54 & 44 & 51 \\
\hline NAR & 158 & 172 & 169 & 125 & 129 \\
\hline
\end{tabular}

TABLE IV

RESULTS OF COMBINATIONS WITH NAR, TISD, FAS IN PROCEDURE 3

\begin{tabular}{|c|c|c|c|c|}
\hline OTAP & MAP & FAAP & TAP & TSAP \\
\hline 212 & 237 & 219 & 185 & 147 \\
\hline
\end{tabular}

\section{Schedules}

1) Fast Annealing Schedule (FAS): The following schedule is used in [12]'s fast annealing.

$$
T_{i}=\frac{T_{\text {init }}}{i}
$$

2) Exponential Schedule (ES): An exponential schedule is by far the most commonly used schedule.

$$
T_{i+1}=\alpha T_{i} \quad \alpha \in(0,1]
$$

3) Geman Schedule (GS): Geman [16] introduced a schedule and they guaranteed convergence to the optimal solution.

$$
T_{i}=\frac{d}{\ln (i+1)} \quad P>0
$$

\section{Parameters Selected Manually}

In a square with sides of 1 kilometer, WS devices are deployed randomly on a plane. The coordinations $(x, y)$ of WS devices are used to calculate the distances $d_{i j}$ between them. In this section, the methods of Neighbor Allocation, Temperature Initialization, Acceptance Rule and Schedule are chosen manually. However, $n$ (steps) and $r$ (Ratio) in Temperature Initialization, $q$ in TSAP and OTAP, $\alpha$ in ES and $d$ in GS are initialized with constants.

Each time 50 devices are deployed randomly. Then SA with all parameters combinations is applied to solve the problem. It repeats 1000 times. The combination of parameters which performs best is recorded every time. How many times of 1000 the combinations perform best is shown in Table I and Table II. Except the combinations in Table I and II, the numbers of times other combinations perform best are 0 . In Table I, the combinations are ES, NAR, different methods of Temperature Initialization and different methods of Acceptance Rule. In Table II, the combinations are FAS, TISD, different methods of Neighbor Allocation and Acceptance Rule.

By analyzing the results of Tables I and II, the combination which performs best will be chosen. At first, the sums of the two tables are calculated respectively. The sum of the times of the combinations with NAR and ES in Table I is 368. The sum of the times of the combinations with TISD and FAS in Table II is 632. Hence, TISD and FAS are chosen to be used as the methods of Temperature Initialization and Schedule respectively.

Next, after TISD and FAS are chosen, the combinations of different methods of Neighbor Allocation and Acceptance Rule repeat 1000 times again. Each time, The combination of parameters which performs best is recorded too. Seen from Table III, the sum of the combinations with NAASA is 247. The sum of the combinations with NAR is 753. Hence, NAR is chosen as the method of Neighbor Allocation.

At last, the combinations with TISD, FAS, NAR and different methods of Acceptance Rule repeat 1000 times again. Seen from Table IV, the largest number of the times that the combination performs best is 237. So MAP is chosen. Hence, NAR, TISD, MAP and FAS are chosen manually as the parameters of SA applied to the problem of channel allocation in TVWS systems.

\section{Parameters Selected using SA as Meta ALGORITHM}

In this section, SA is used to choose the parameters of SA automatically. The states in the high level SA are the combinations of parameters of the low level SA. The solution space is the domain of all possible combinations. The parameters that will be chosen include Neighbor Allocation, Temperature Initialization, Acceptance Rule and Schedule. Furthermore, $n$ (steps) and $r$ (Ratio) in Temperature Initialization, $q$ in TSAP and OTAP, $\alpha$ in ES and $d$ in GS, which are fixed in the procedure of manual selection, are also chosen.

The flow chart of the algorithm using SA as meta algorithm is shown in Fig. 3. The parameters of high level SA are classic. Temperature is initialized with a constant. Neighbor combination is selected at random. $e^{-\triangle F / T}$ is used as acceptance rule. $T=T_{0} / \log (i)$ is used as schedule. In Fig. 3, after generating a new combination, the new combination is used to optimize channel allocation 50 times. Every time, fitness $F$ is computed. In the end, the standard deviation of all the 50 fitnesses is used as $F_{\text {new }}$ to avoid accidental factors.

After running the algorithm, the combination selected is \{NAR, TISD, 0.9734, 1069, FAAP and FAS $\}$. In the results, 0.9734 and 1069 are ratio and steps used in TISD. Except Acceptance Rule, other parameters are the same as the parameters selected manually. To see which one is better, classic SA and SA with the parameters selected automatically and manually are applied to optimize channel allocation respectively. Seen from Fig.4, SA with the parameters selected automatically performs best. 


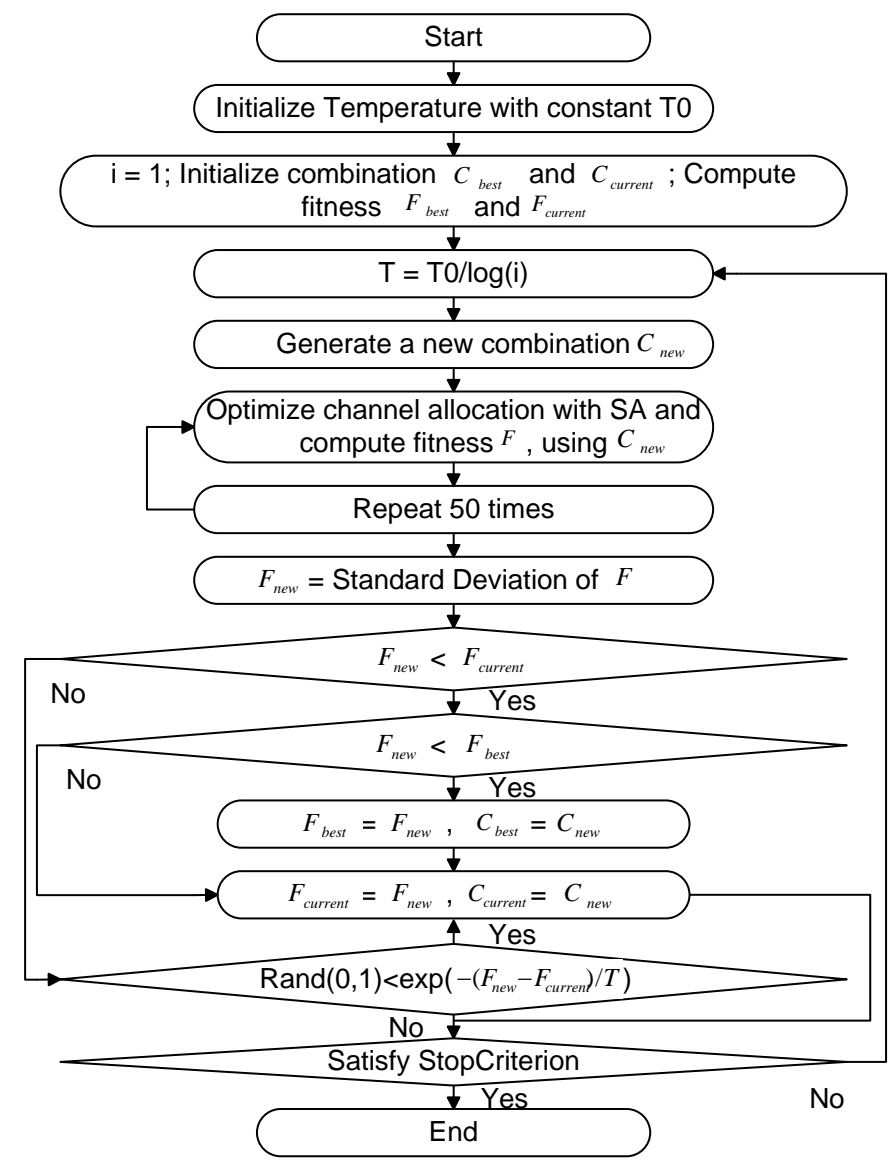

Fig. 3. Flow Chart of SA with SA as Meta Algorithm

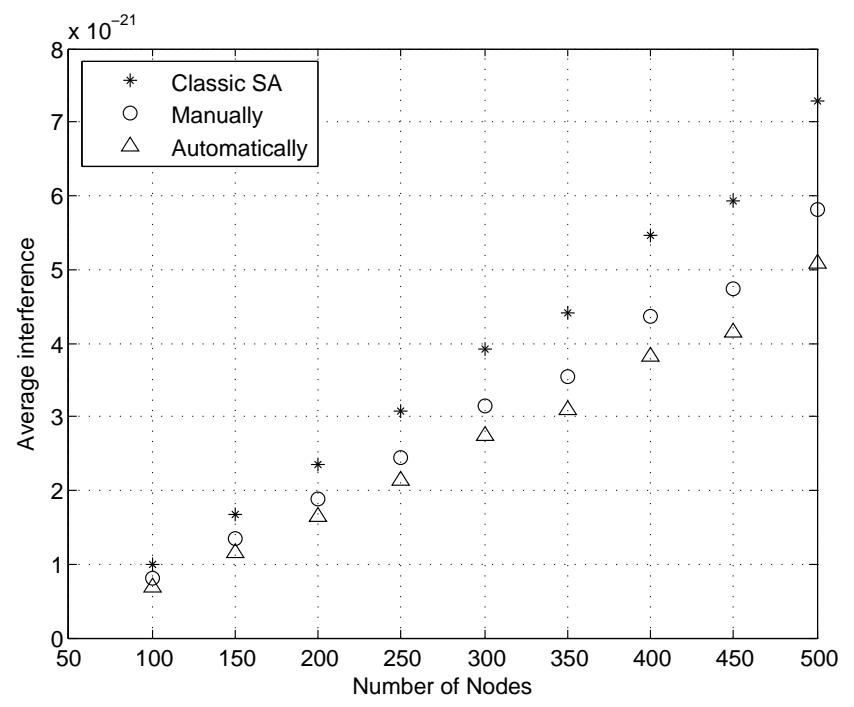

Fig. 4. Channel Allocation Using Classic SA and SA with the Parameters Selected Automatically, Manually

\section{CONCLUSION}

In this paper, the application of SA to solve channel allocation problem is introduced in TVWS systems. Details of SAs parameters have been given. Extensive analysis and exper- imental simulations have been carried out to provide an insight on how to choose various parameters to apply the optimization strategy. This paper can give some guidance to the choice of various parameters of SA which are applied to channel allocation problem in TVWS systems. Moreover it helps select parameters automatically with SA as meta algorithm. The most parameters of the combination selected manually and automatically are the same. But, in the manual procedure, some parameters are fixed due to its limitations while they are selected automatically in the automatic procedure. Therefore, the parameters selected automatically performs better than parameters selected manually.

\section{REFERENCES}

[1] J. Y. Chen, S. Olafsson, X. Y. Gu and Y. Yang, A fast channel allocation scheme using simulated annealing in scalable WLANs. Broadband Communications, Networks and Systems, 2008. BROADNETS 2008. 5th International Conference on, pp. 205.

[2] M. Asvial, B.G. Evans, and R. Tafazolli, Evolutionary genetic DCA for resource management in mobile satellite systems. Electronics Letters, vol. 38, no. 20, pp. 1213-1214, 2002.

[3] I. E. Kassotakis, M. E. Markaki, A hybrid genetic approach for channel reuse in multiple access telecommunication networks. Selected Areas in Communications, IEEE Journal on, vol. 18, no. 2, pp. 234-243, 2000.

[4] S. S. M. Patra, K. Roy, S. Banerjee and D. P. Vidyarthi, Improved genetic algorithm for channel allocation with channel borrowing in mobile computing. Mobile Computing, IEEE Transactions on, vol. 5, no. 7, pp. 884-892, 2006.

[5] A. Y. Zomaya and M. Wright, Observations on using genetic-algorithms for channel allocation in mobile computing. Parallel and Distributed Systems, IEEE Transactions on, vol. 13, no. 9, pp. 948-962, 2002.

[6] D. J. Leith and P. Clifford, A Self-Managed Distributed Channel Selection Algorithm for WLANs. Modeling and Optimization in Mobile, Ad Hoc and Wireless Networks, 4th International Symposium on, pp. 1, 2006.

[7] D. Kunz, Channel assignment for cellular radio using neural networks. Vehicular Technology, IEEE Transactions on, vol. 40, pp. 188-193, 1991.

[8] J. Y. Chen, S. Olafsson, and X.Y. Gu, A Biologically Inspired Dynamic Channel Allocation Technique in 802.11 WLANs with Multiple Access Points. Personal, Indoor and Mobile Radio Communications, 2007. PIMRC 2007. IEEE 18th International Symposium on, pp. 1.

[9] Ofcom, Statement on Cognitive Access to Interleaved Spectrum. July 1, 2009; http://www.ofcom.org.uk/consult/condocs/cognitive/statement.

[10] L. Ingber, Very Fast Simulated Re-Annealing. Mathematical and Computer Modelling, 12, 967-973, 1989.

[11] S. R. White, Concepts of Scale in Simulated Annealing. In IEEE Proceedings of the 1984 International Conference on Computer Design, pp. 646-651, 1984.

[12] H. Szu and R. Hartley, Fast simulated annealing. Physics Letters A, Volume 122, Issues 3-4, pp. 157-162, 1987.

[13] C. Tsallis and D. A. Stariolo, Generalized simulated annealing. Physica A 233, pp. 395-406, 1996.

[14] A. Franz and K. H. Hoffmann, Threshold accepting as limit case for a modified Tsallis statistics. Applied Mathematics Letters, Volume 16, Issue 1, pp. 27-31, 2003.

[15] G. Dueck and T. Scheuer, Threshold accepting: A general purpose optimization algorithm appearing superior to simulated annealing. Journal of Computational Physics, Volume 90, Issue 1, pp. 161-175, 1990.

[16] S. Geman and D. Geman, Stochastic Relaxation, Gibbs Distributions, and the Bayesian Restoration of Images. In Proceedings of Sixth IEEE Pattern Analysis and Machine Intelligence, pp. 721-741, 1984. 